\title{
Cluster of cases of pulmonary melioidosis presenting as acute fulminant pneumonia in the East of Sri Lanka
}

Jayaweera $\mathbf{L J}^{1}$, Wickramasinghe $\mathbf{D}^{2}$, Randeep $\mathrm{K}^{1}$, Wijerathna $\mathbf{P}^{2}$, Javahir $\mathbf{I M}^{1}$, Sharjoon $\mathbf{M}^{1}$, Ismail $\mathbf{S}^{1}$, Rajesegram $\mathbf{J}^{1}$, Liyanage $\mathrm{SS}^{1}$, Dias $\mathrm{ND}^{1}$, Corea $\mathrm{EM}^{3}$

Journal of the Ceylon College of Physicians, 2021, 52, 34-39

\begin{abstract}
Melioidosis is a potentially fatal infection caused by the soil-associated, Gram negative bacterium Burkholderia pseudomallei. It is still largely under recognized as a cause of community acquired, acute fulminant pneumonia.

We identified 16 patients from a single rural village in the Eastern Province of Sri Lanka who were admitted with pulmonary melioidosis during a fourmonth period (October 2017 to February 2018). They presented with fever, cough, and shortness of breath for 3-5 days. Chest radiographs showed bilateral multiple pulmonary nodules with cavitation. Diagnosis was confirmed by positive blood cultures and/or rising antibody titers. The unique features of this cluster were the female predominance and asthma being the predominant risk factor. Epidemiological observations suggest that host behavior and prevailing weather conditions may have triggered this cluster of pulmonary melioidosis. Hence, melioidosis should be considered in the differential diagnosis of patients with severe community acquired pneumonia in the relevant epidemiological setting.
\end{abstract}

Key words: pulmonary melioidosis, case cluster/ outbreak, Burkholderia pseudomallei, asthma, Sri Lanka

\section{Introduction}

Melioidosis is endemic in certain parts of the world, such as Southeast Asia and Northern Australia causing a significant number of community-acquired pneumonia and sepsis., ${ }^{1,2}$ Melioidosis has protean clinical manifestations due to its ability to affect multiple organs in the body or causing bacteraemia. ${ }^{3}$ Pulmonary disease is recognized as the commonest clinical manifestation. ${ }^{3}$ Pathological changes seen in pulmonary melioidosis can give rise to varying appearances on chest radiographs. Acute pneumonia may manifest as multiple small pulmonary nodules and multi lobar infiltrations starting from the upper lobes. As the disease progresses, they can form multiple cavities and abscesses. ${ }^{4,5}$ Such changes may mimic staphylococcal infection. Sub-acute and chronic forms with patchy nodular opacities may mimic tuberculosis.

Diabetes mellitus is the commonest underlying predisposing condition for melioidosis ${ }^{6,7}$ and prevalent in $63 \%$ of the culture positive patients recorded in the Sri Lankan national melioidosis surveillance data. ${ }^{8}$

The definitive diagnosis of melioidosis is made by isolation of $B$. pseudomallei from biological samples. Demonstration of antibodies in a patient is suggestive of infection but not confirmatory due to the presence of seropositive healthy individuals in endemic areas. $^{9,10}$ Melioidosis has been compared with an iceberg due to under recognition of most cases. ${ }^{11}$ In Sri Lanka, too, B. pseudomallei is still not appreciated

${ }^{1}$ Akkaraipattu Base Hospital, Sri Lanka, ${ }^{2}$ District General Hospital, Ampara, Sri Lanka, ${ }^{3}$ Department of Microbiology, Faculty of Medicine, University of Colombo, Sri Lanka.

Correspondence: LJJ, e-mail: ljjayaweera@gmail.com

iD https://orcid.org/0000-0001-9822-0893

Received 18 April 2021, accepted 21 May 2021.

This is an open-access article distributed under the terms of the Creative Commons Attribution License, which permits unrestricted use, distribution, and reproduction in any medium, provided the original author and source are credited. 
as a leading cause of community acquired infection due to lack of awareness among clinicians and limited laboratory facilities in rural regions. ${ }^{12}$ National surveillance for melioidosis has identified a wide distribution of cases across the country. ${ }^{8}$ However, there is no established notification or reporting system locally for melioidosis.

\section{Case series}

Kolavil is a small coastal village consisting of paddy lands, wetlands and grasslands situated in the Ampara District of the Eastern Province of Sri Lanka. The wetlands are situated to the east of the village abutting a lagoon (Figure 1). Approximately 5377 people live in Kolavil (Resource profile of Kachcheri, Ampara Sri Lanka) and the major livelihoods of the area are fisheries, rice and vegetable cultivation, trade and government and non-governmental services. A few families engage in livestock farming, mainly cows and goats. Rainfall varies seasonally in this region, where the northeast monsoon (December-February) gives the highest rainfall followed by the second inter-monsoon (October-November). ${ }^{13}$ Villagers reported excavating

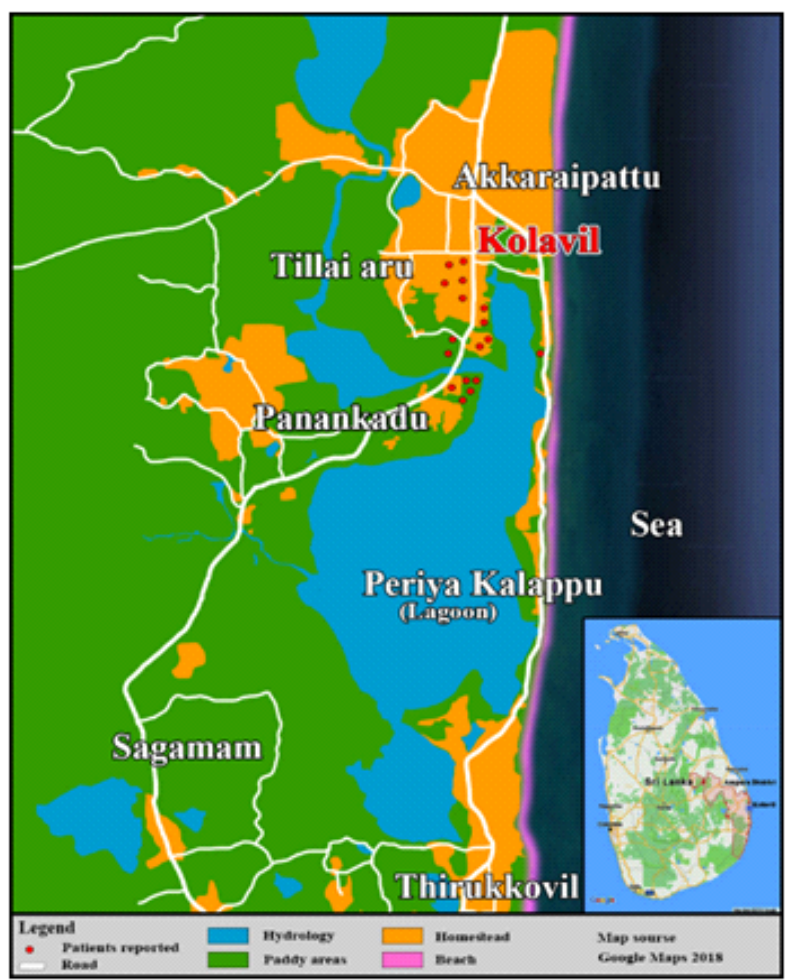

Figure 1. Map of Kolavil showing the geographic distribution of the 16 cases of melioidosis.

Map source - https://landsatlook.usgs.gov/sentinel2/ viewer.html soil from a land abutting an old grave-yard to 'sand' their gardens prior to the northeast monsoon in 2017 , an annual practice done to prevent flooding.

Sixteen inhabitants or visitors to Kolavil were diagnosed with melioidosis between October 2017 and February 2018 (Table 1). Diagnosis was based on clinical features of acute onset community acquired pneumonia (CAP), evidence of alveolar infiltrates on chest radiographs, rising or falling antibody titre on indirect haemagglutination assay (IHA) and /or culture positivity $(n=5)$. There were more females $(n=10)$ than males $(n=6)$. Age range was from 18 to 74 years with a median of 53 years. Eleven (68\%) patients had a history of asthma, among whom five also had diabetes mellitus. Five were previously healthy. Four patients succumbed to the disease. Case histories of three patients are presented in detail to highlight the challenges in diagnosing and managing a case cluster of melioidosis in a rural community.

\section{Case 1}

A 27-year-old woman was admitted with a four-day history of fever, cough, and shortness of breath. She was known to have mild intermittent asthma. On physical examination she had a temperature of $38.8^{\circ} \mathrm{C}$, pulse rate of 110 beats per minute and respiratory rate of 36 breaths per minute. Oxygen saturation was $88 \%$ and $\mathrm{PaO}_{2}$ was $76 \mathrm{mmHg}$ while on $40 \%$ inspired oxygen. Her initial chest radiograph was normal. Investigations revealed, leukocyte count of $12.4 \times 10^{9} / \mathrm{L}$ with $82 \%$ neutrophils and C-reactive protein (CRP) of $10 \mathrm{mg} / \mathrm{dL}$. Initial blood and sputum cultures were negative. Treatment was commenced for community acquired pneumonia with intravenous co-amoxiclav $1.2 \mathrm{~g} 8$ hourly and clarithromycin 500mg twice a day. After 4 days her symptoms worsened, and multiple alveolar infiltrates appeared on the chest radiograph (Figure 2A). Co amoxiclav was replaced by ceftriaxone and vancomycin with some improvement in her clinical status. However, on the $12^{\text {th }}$ day of admission she deteriorated with reappearance of fever $\left(39.4^{\circ} \mathrm{C}\right)$ and worsening hypoxaemia $\left(\mathrm{PaO}_{2}\right.$ of $52 \mathrm{~mm} \mathrm{Hg}$ while on $60 \%$ inspired oxygen). The chest radiograph showed diffuse bilateral patchy infiltrates throughout both lung fields (Figure 2B). Despite invasive mechanical ventilation and attempts at resuscitation the patient suffered multiple cardiac arrests and died a day later. Postmortem examination showed multiple pus-filled cavities in the lungs (Figure 3). Blood cultures taken during this period was positive for B. pseudomallei and the melioidosis antibody titre by the IHA was 1: $>10240$. 
Table 1. Melioidosis cases reported in Kolavil from October 2017 to February 2018

*Cases presented in detail

\begin{tabular}{|c|c|c|c|c|c|c|c|c|}
\hline Age & Sex & Occupation & $\begin{array}{c}\text { Underlying } \\
\text { diseases }\end{array}$ & $\begin{array}{c}\text { Neutrophil } \\
\text { Leuko- } \\
\text { cytosis }\end{array}$ & Month & $\begin{array}{c}\text { IHA values } \\
\text { (on admission } \\
\text { and the } \\
\text { highest value) }\end{array}$ & $\begin{array}{l}\text { Microbiology- } \\
\text { Blood culture }\end{array}$ & Outcome \\
\hline 55 & $M$ & Farmer & BA, DM, HTN & + & October & & Culture + & Died \\
\hline $27^{*}$ & $\mathrm{~F}$ & Housewife & $\mathrm{BA}$ & + & November & 1:160; 1:10240 & Culture+ & Died \\
\hline 73 & $\mathrm{~F}$ & Housewife & BA, DM & + & November & $1: 2560,1: 160$ & Culture+ & Recovered \\
\hline 37 & $\mathrm{~F}$ & Housewife & $\mathrm{BA}$ & + & November & $1: 160,1: 1280$ & & Recovered \\
\hline $18^{*}$ & $\mathrm{~F}$ & Student & $\mathrm{BA}$ & + & December & & Culture+ & Died \\
\hline 35 & $\mathrm{~F}$ & Housewife & BA & + & December & $1: 80,1: 320$ & & Recovered \\
\hline 60 & $M$ & Gardener & $\mathrm{BA}, \mathrm{DM}, \mathrm{HTN}$ & + & December & $1: 80,1: 320$ & & Recovered \\
\hline 38 & $\mathrm{~F}$ & Housewife & & + & December & $1: 80$ & & Recovered \\
\hline $60^{*}$ & $\mathrm{~F}$ & Farmer & & + & December & $1: 1240 ; 1: 10240$ & & Recovered \\
\hline 58 & $\mathrm{~F}$ & Housewife & RA, DM, BA & + & December & $1: 160,1: 40$ & & Recovered \\
\hline 37 & $\mathrm{~F}$ & Housewife & & + & December & $1: 40,1: 80,1: 320$ & & Recovered \\
\hline 70 & M & Farmer & & + & December & $1: 20$ & Culture + & STEMI; Died \\
\hline 42 & $\mathrm{~F}$ & Housewife & $\mathrm{BA}, \mathrm{DM}$ & + & January & $\begin{array}{l}\text { Negative; } \\
\text { 1:2560, } \\
1:>10240\end{array}$ & & Recovered \\
\hline 72 & M & Farmer & $\mathrm{BA}$ & + & January & $1: 80,1: 160$ & & Recovered \\
\hline 74 & M & Farmer & & + & February & $1: 160,1: 40$ & & Recovered \\
\hline 57 & $M$ & Painter & $\mathrm{BA}$ & + & February & 1:640,1:320 & & Recovered \\
\hline
\end{tabular}

(M; Male, F; Female, BA; bronchial asthma, RA; rheumatoid arthritis, DM; diabetes mellitus, HTN; hypertension, IHA; indirect haemagglutination assay, Culture +; Burkholderia pseudomallei isolated in blood culture, STEMI; ST elevated myocardial infarction)

\section{Case 2}

An 18-year-old woman with a history of asthma was admitted with severe shortness of breath. She had a history of fever, cough and wheezing for 4 days and had been treated at the primary care level with antibiotics. On admission, she was in septic shock and type 1 respiratory failure. Arterial blood gas showed metabolic acidosis with hypoxia $\left(\mathrm{pH} 7.14, \mathrm{HCO}_{3} 8.9\right.$
$\mathrm{mEq} / \mathrm{L}, \mathrm{PaCO}_{2} 24 \mathrm{mmHg}, \mathrm{PaO}_{2} 72 \mathrm{mmHg}$ while on $60 \%$ oxygen). Her chest radiograph showed bilateral widespread patchy opacities. Sepsis management was commenced immediately with fluid resuscitation, and intravenous ceftazidime was given considering the high probability of melioidosis. She was intubated and started on inotropes, but she died within one hour of hospital admission. Blood cultures were reported as positive for B. pseudomallei. 

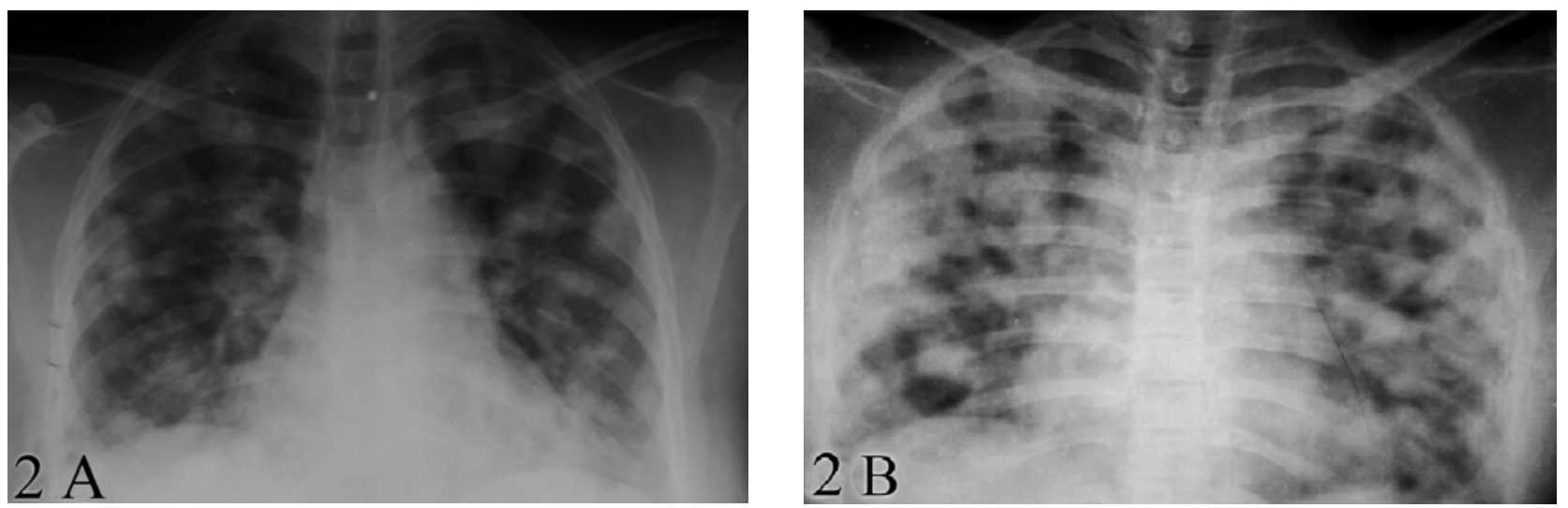

Figure 2. Chest radiography showing progression of patchy opacities (2A \& 2B).
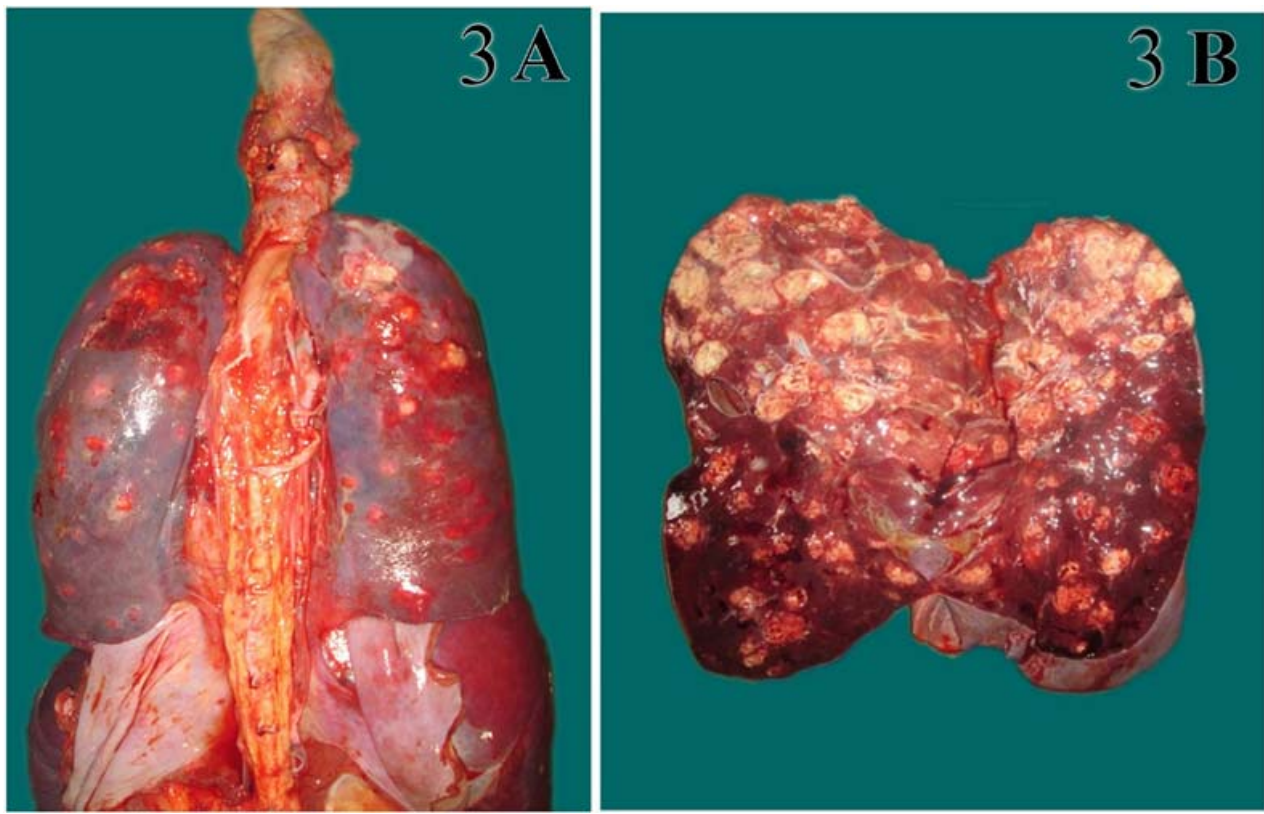

Figure 3. Case 1 - Post mortem findings of lungs. Outer surface of both lungs show numerous whitish nodules involving all lobes (3A). Cut surface shows congested lung parenchyma with multiple discrete white solid nodules with central cavitation (3B).

\section{Case 3}

A 60-year-old female farmer with a history of asthma was admitted with a five-day history of fever, cough, and wheezing. On admission her temperature was $38.8^{\circ} \mathrm{C}$, blood pressure was $100 / 80 \mathrm{~mm} \mathrm{Hg}$, heart rate was $96 \mathrm{bpm}$, respiratory rate was 48 breaths per minute and oxygen saturation was $94 \%$ on room air. There were rhonchi in both lung fields. Initial chest radiograph showed a cavitating opacity on the right upper zone with a few areas of consolidation on the left side (Figure 4). Empirical treatment was started with intra-venous ceftazidime (2g 8 hourly) and oral co-trimoxazole (960mg twice a day). Blood cultures were negative. Initial melioidosis titre was 1:1240, which rose to more than $1: 10,240$ in two weeks. She recovered completely in three weeks with the antibody titre dropping to $1: 80$. 


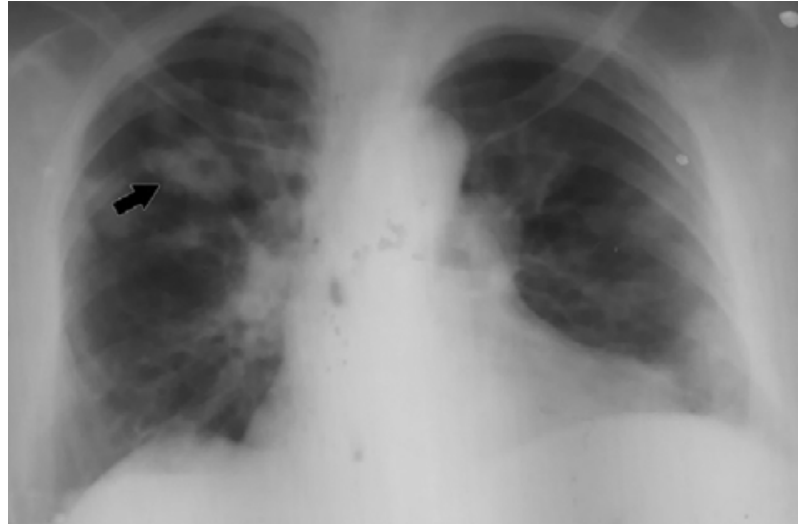

Figure 4. Chest radiograph of Case 3 showing a cavitary lesion on the right upper lobe (arrow) with bilateral air space consolidation.

\section{Discussion}

Melioidosis is usually a sporadic infection. Outbreaks or case clusters are rare and usually reflect an unusual convergence of environmental factors. ${ }^{14,15}$ The case cluster described in this paper gives an insight into previously unrecognized environmental and host determinants of infection.

It is known that the incidence of melioidosis increases in the wettest months of the year. ${ }^{7}$ Our case cluster took place in October to February, which is the monsoon period for the East of the country. The heaviest rain fall was recorded in mid-November. ${ }^{13}$ The speed of the gusts in the area steadily rose from early October and peaked in mid-November. ${ }^{13}$ We suspect that digging of the sand for 'sanding' of the gardens brought up the deeper soil containing the organism to the surface and the loose soil was blown around the area with the monsoon gusts increasing airborne transmission. Environmental sampling methods were not available in Sri Lanka to confirm the source of infection.

Although melioidosis can affect any organ of the body, all patients in this case cluster presented as severe community acquired pneumonia (CAP) with acute onset of fever, cough, and shortness of breath. ${ }^{16}$ This may be due to the probable mode of acquisition being inhalation of contaminated sand aerosolized by high winds. An unusual feature observed in our case cluster was that asthma was the predominant underlying disease. The clinical course was rapid necessitating admission to hospital within 5 days of onset. Presentation was similar in all cases with pyrexia $\left(>38.8^{\circ} \mathrm{C}\right)$, tachycardia and tachypnoea, crackles and rhonchi on chest auscultation, a raised
CRP level and neutrophil leukocytosis. Fifteen of the sixteen patients had changes in the chest radiograph on admission. All had patchy consolidation and /or nodules distributed in multiple lung zones. In six patients, the initial radiographs showed involvement of the right upper lobe with cavities and nodules mimicking pulmonary tuberculosis. Pleural effusions or lung abscesses were not seen in our patients. All the patients who recovered showed resolution of lung opacities in the chest radiographs by the end of the third week of treatment.

Many studies have shown that pulmonary melioidosis is difficult to differentiate from other causes of severe CAP. ${ }^{17,18}$ The conventional antibiotics recommended for the treatment of CAP and sepsis are not effective in melioidosis. ${ }^{19,20}$ Antibiotic guidelines recommend ceftazidime or meropenem for induction therapy and co-trimoxazole for eradication phase following laboratory diagnosis of melioidosis. ${ }^{19,20}$ Once we became aware of the epidemic, residents of Kolavil admitted with severe CAP were started on specific melioidosis therapy with better outcomes.

It is difficult and time consuming to obtain confirmatory laboratory evidence of melioidosis, especially in resource limited settings. The sensitivity of culture in melioidosis ranges from $51.7 \%-68.5 \% .^{21}$ In this case series the blood culture positivity rate was as low as $31 \%(5 / 16)$ and none of the sputum cultures were positive. This could be due to prior antibiotic treatment, low volume of blood taken for culture, lack of an automated blood culture system and low levels of bacteraemia. The four of the five-blood culture positive patients succumbed to the infection, probably due to high levels of bacteraemia and overwhelming sepsis. Further, melioidosis antibodies by the IHA test may not be positive in acute cases as there is inadequate time to mount an immune response. Therefore, absence of a positive culture or antibody test does not exclude melioidosis, and a high index of clinical suspicion is needed in patients with typical clinical and radiological features in the correct epidemiological setting.

Melioidosis is not a notifiable disease in Sri Lanka. Lack of a coordinated notification system for melioidosis was a barrier to prompt reporting and dissemination on information.

Despite several patients from the same village had already been diagnosed with melioidosis, the local general practitioners and the community were not aware of this. Subsequently, we reported all suspected patients to the Regional Epidemiologist and Public Health Inspectors who in turn, informed the villagers 
to seek immediate medical attention in case of fever and cough. This resulted in earlier hospitalizations and better outcome in the subsequent cases.

\section{Conclusion}

Melioidosis is under recognized as a leading cause of life threatening, acute fulminant pneumonia in endemic countries. Early detection, treatment and notification can help to minimize mortality. Lack of diagnostic facilities and sharing of epidemiological data in rural areas hampers early identification of patients. Public health authorities should urgently focus on measures to enhance melioidosis surveillance, notification and environmental investigations at the local level. This paper highlights the importance of setting up a local melioidosis surveillance and notification system to disseminate such information to clinicians and to take prompt public health actions.

\section{Acknowledgements}

We thank Dr. Gunarathna S.W.A.U., Dr.Thilshan M.N.M., Dr. Inshad M.I.M., Dr. Ajihar M.A.M., the intern house officers of Medical Unit ABH, Sri Lanka, who were involved in the management of these patients and Dr W. Dissanayaka, Consultant Pathologist, for her help in the pathological examination.

\section{Conflicts of interests}

There are no conflicts of interests.

\section{References}

1. Chaowagul W, White NJ, Dance DA, Wattanagoon $\mathrm{Y}$, Naigowit P, Davis TM, et al. Melioidosis: a major cause of community-acquired septicemia in northeastern Thailand. $J$ Infect Dis. 1989: 159; 890-9.

2. Currie BJ, Ward L, Cheng AC. The Epidemiology and Clinical Spectrum of Melioidosis: 540 Cases from the 20 Year Darwin Prospective Study. PLoS Negl Trop Dis. 2010; 4(11): e900. https://doi.org/10.1371/journal.pntd.0000900

3. Dance D. Melioidosis as an emerging global problem. Acta Tropica 2000; 74(2-3): 115- 119. https://doi.org/10.1016/ S0001-706X(99)00059-5

4. Muttarak M, Peh W, Euathrongchit J, et al. Spectrum of imaging findings in melioidosis. Br J Radiol. 2009; 82(978): 514-21.

5. Dhiensiri T, Puapairoj S, Susaengrat W. Pulmonary melioidosis: clinical-radiologic correlation in 183 cases in northeastern Thailand. Radiology 1988; 166: 711-15.
6. Suputtamongkol $\mathrm{Y}$, Chaowagul W, Chetchotisakd $\mathrm{P}$, et al. Risk Factors for Melioidosis and Bacteremic Melioidosis. Clin Infect Dis.1999; 29(2): 408-13.

7. Hassan M, Pani S, Peng N, et al. Incidence, risk factors and clinical epidemiology of melioidosis: a complex socioecological emerging infectious disease in the Alor Setar region of Kedah, Malaysia. BMC Infectious Diseases 2010: 10 : 302. https://doi.org/10.1186/1471-2334-10-302

8. Corea E, Ler Y, Thevanesam V, Merritt A, Inglis, T. Sri Lankan National Melioidosis Surveillance Program Uncovers a Nationwide Distribution of Invasive melioidosis. Am J Trop Med Hyg. 2016; 94(2) : 292-8.

9. Zysk G, Splettstosser WD, Neubauer H. A review on melioidosis with special respect on molecular and immunological diagnostic techniques. Clin Lab. 2000; 46: 119-30.

10. Sirisinha S, Anuntagool N, Dharakul T, et al. Recent developments in laboratory diagnosis of melioidosis. Acta Trop. 2000; 74: 235-45.

11. Dance DA. Melioidosis; the tip of the iceberg? Clin Microbiol Rev. 1991; 4(1): 52-60.

12. Corea EM, de Silva AD, Thevanesam V. Melioidosis in Sri Lanka. Trop. Med. Infect. Dis. 2018; 3(1): 22.

13. www.worldweatheronline.com/ampara-weatheraverages/eastern-province/k.aspx. Accessed 12 May 2021

14. Inglis T, O'Reilly L, MerrittA, Levy A, Heath C. The Aftermath of the Western Australian Melioidosis Outbreak. Am J Trop Med Hyg. 2011; 84(6): 851-7.

15. Ko WC, Cheung BM, Tang HJ, Shih HI, Lau YJ, Wang LR, Chuang YC. Melioidosis outbreak after typhoon, southern Taiwan. Emerg Infect Dis. 2007; 13(6): 896-8.

16. Meumann EM, Cheng AC, Ward L, Currie BJ. Clinical features and epidemiology of melioidosis pneumonia: results from a 21-year study and review of the literature. Clin Infect Dis. 2012; 54: 362-9.

17. Currie B J. Melioidosis: an important cause of pneumonia in residents of and travelers returned from endemic regions, Eur Respir J. 2003; 22: 542-50; doi: 10.1183/ 09031936.03.00006203

18. White N, Chaowagul W, Wuthiekanun V, et al. Halving of Mortality of Severe Melioidosis by Ceftazidime. The Lancet. 1989; 334(8665): 697-701.

19. Antibiotic Guidelines 2016 | Sri Lanka College of Microbiologists . http://slmicrobiology.net/antibiotic-guidelines2016, Accessed 15 May 2021

20. Inglis T. The Treatment of Melioidosis. Pharmaceuticals 2010; 3(5): 1296-303.

21. Limmathurotsakul D, Jamsen $K$, Arayawichanont $A$, et al. Defining the True Sensitivity of Culture for the Diagnosis of Melioidosis Using Bayesian Latent Class Models. PLOS ONE. 2010; 5(8): e12485. https://doi.org/10.1371/ journal.pone.0012485 